\title{
Optimal multiqubit operations for Josephson charge qubits
}

\author{
Antti O. Niskanen* Juha J. Vartiainen đ and Martti M. Salomaa \\ Materials Physics Laboratory, POB 2200 (Technical Physics), \\ FIN-02015 HUT, Helsinki University of Technology, Finland
}

(Dated: November 18, 2018)

\begin{abstract}
We introduce a method for finding the required control parameters for a quantum computer that yields the desired quantum algorithm without invoking elementary gates. We concentrate on the Josephson charge-qubit model, but the scenario is readily extended to other physical realizations. Our strategy is to numerically find any desired double- or triple-qubit gate. The motivation is the need to significantly accelerate quantum algorithms in order to fight decoherence.
\end{abstract}

PACS numbers: 03.67.Lx, 03.75.Lm, 02.60.Pn

Keywords: quantum computation, Josephson effect, numerical optimization

Quantum computing algorithms are realized through unitary operators that result from the temporal evolution of the quantum system under consideration. Typically, these are achieved with a sequence of universal gates [1] which act analogously to the elementary gates of digital computers. Quantum computers hold the promise of exponential speedup with respect to classical computers owing to the massive parallelism arising from the superposition of quantum bits, qubits; for introductions to quantum computing and quantum information processing, see Refs. 2]. Several different physical implementations of quantum computing have been suggested; in particular quantum computing with Cooper pairs has been proposed [3].

Superconducting circuits [4] feature controlled fabrication and scalability [5]; their drawback is that the leads inevitably couple the qubit to the environment, thereby introducing decoherence [6]. In a superconductor, the number of the Cooper pairs and the phase of the wavefunction constitute conjugate variables. The majority of investigations has focused either on the charge regime where the number of Cooper pairs is well defined [7], or on the flux regime where the phase is well defined [8]. Qubits utilizing current-driven large Josephson junctions have been tested experimentally $9 \mid$. The decisive experimental progress reported in Ref. 10] has demonstrated that it is possible to realize $10^{4}$ elementary quantum gates with Josephson-junction qubits. Here we consider Josephson charge qubits.

In this Letter we propose a method to construct arbitrary two- or three-qubit quantum gates by solving the numerical optimization problem of control parameters for a Josephson charge qubit register. We show that it is possible to numerically find the required control-parameter sequences even for nontrivial three-qubit gates without employing elementary gates. Recently, it has been suggested [11] how to solve a similar problem in the context of holonomic quantum computation [12], where time does not appear as an explicit parameter. In the present context, the time evolution appears through the Schrödinger equation.

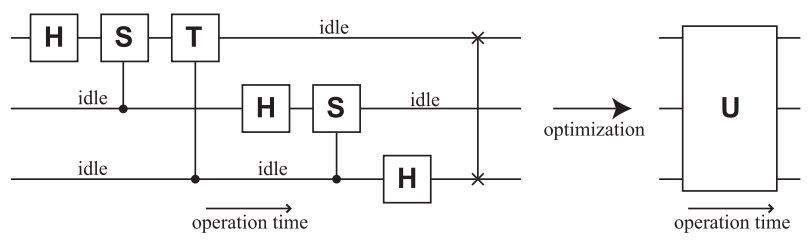

FIG. 1: Instead of implementing the three-qubit quantum Fourier transform with the help of elementary gates, we determine a gate that performs the entire three-qubit operation with a single control loop. Note that idle time is avoided.

The motivation underlying the investigation of this approach is the need to overcome effects of decoherence. The implementation of a quantum algorithm which is composed of elementary gates is rarely optimal in execution time since the majority of qubits is most of the time inactive, see Fig. [1 The decomposition into elementary gates works extremely well with classical digital computers. However, in the context of quantum computing the number of consecutive operations is strictly limited by the short time window set by interactions with the environment. It is therefore of prime importance to concentrate on the implementations of quantum algorithms 13, 14, 15. We consider the construction of quantum algorithms out of larger building blocks. Whereas careful design and manufacturing can significantly increase the decoherence time, our scenario can serve to reduce the number of the operations needed.

The Josephson charge qubit utilizes the number degree of freedom of a nanoscale Josephson-junction circuit. The states of the qubit correspond to either zero or one extra Cooper pair residing on the superconducting island, usually denoted by $|0\rangle$ and $|1\rangle$, respectively. The Cooper pairs can tunnel coherently to a superconducting electrode. The charging energy of the qubit can be tuned with the help of an external gate voltage, whereas tunneling between the states is controlled with the help of an external magnetic flux.

The explicit single-qubit Hamiltonian for the qubit $i$ is 


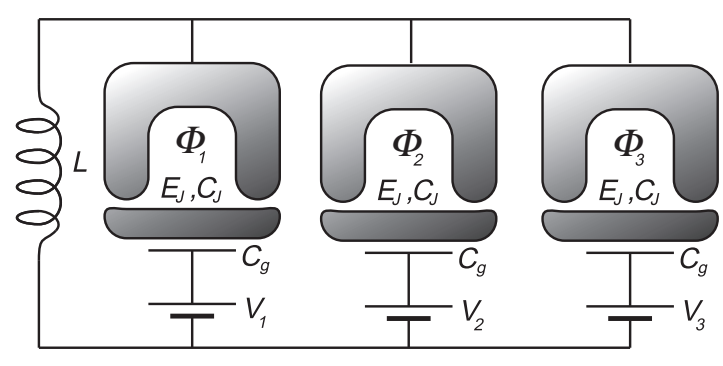

FIG. 2: Schematic illustration of three Josephson charge qubits with inductive coupling. The adjustable parameters include the gate voltages $V_{i}$ and the enclosed fluxes $\Phi_{i}$.

$$
H_{\text {single }}^{i}=-\frac{1}{2} B_{z}^{i} \sigma_{z}-\frac{1}{2} B_{x}^{i} \sigma_{x}
$$

where the standard notation for Pauli matrices has been utilized. Here $B_{z}^{i}$ is a tunable parameter which depends on the gate voltage, while $B_{x}^{i}$ can be controlled with the help of a flux through the SQUID. Note that setting $B_{z}^{i}=$ $B_{x}^{i}=0$ results in degeneracy. At the degeneracy point, there will be no change in the physical state of the system. In the case of single-qubit gates, it is easy to see from this model that any rotation in $S U(2)$ can be performed on the qubits. Note that $U(2)$ is not available since the Hamiltonian is traceless. In general, we cannot achieve $U\left(2^{N}\right)$ for $N$ qubits since the Hamiltonian of the entire quantum register turns out to be traceless. However, the global phase factor is not physical since it corresponds to a redefinition of the zero level of energy.

Qubits can be coupled by connecting them in parallel to an inductor, see Fig. 22 This scenario has the benefit of allowing for a longer decoherence time and that of being tunable. The resulting coupling term in the Hamiltonian between the qubits $i$ and $j$ is then of the form

$$
H_{\text {coupling }}=-C B_{x}^{i} B_{x}^{j} \sigma_{y} \otimes \sigma_{y}
$$

where $C$ is a positive parameter depending on the capacitances of the qubits and also on the inductance. It follows from Eqs. (12) that one can apply nontrivial two-qubit operations by simultaneously turning on the SQUIDs of the two qubits, although the $\sigma_{x}$-term will be turned on as well. All the other qubits must have their SQUIDs turned off. On the other hand, one-qubit $\sigma_{x^{-}}$ operations require that all but one SQUID is turned on. Note that in the present context it is actually impossible to perform independent operations on any two subsets of the quantum register due to the inductive coupling. Since one must also take into account the decoherence mechanism, it is not practical to let most qubits reside at their degeneracy point. The question arises whether it would rather prove more efficient to try and find some scheme of finding larger quantum operations, instead of using elementary gates.
To tackle the challenge posed above, we concentrate on finding quantum gates numerically. The structure of the Josephson-qubit Hamiltonian is such that it is not immediately transparent how one would actually construct even the basic controlled-NOT gate. We accomplish this by considering loops $\gamma(t)$ in the control-parameter space spanned by $\left\{B_{x}^{j}(t)\right\}$ and $\left\{B_{z}^{j}(t)\right\}$. Therefore, the function $\gamma(t)$ is of the vector form

$$
\gamma(t)=\left[\begin{array}{lllllll}
B_{z}^{1}(t) & \ldots & B_{z}^{N}(t) & B_{x}^{1}(t) & \ldots & B_{x}^{N}(t)
\end{array}\right]^{T},
$$

where we have assumed a register of $N$ qubits. The temporal evolution induces the unitary operator

$$
U=\mathcal{T} \exp \left(-i \int_{\gamma(t)} H(\gamma(t)) d t\right)
$$

where $\mathcal{T}$ stands for the time-ordering operator and we choose $\hbar=1$. The integration is performed along the path formed by $\gamma(t)$ where the loop starts at the origin, i.e., at the degeneracy point. We will restrict the path to a special class of loops, which form polygons in the parameter space. Thus the parameters vary in time at a piecewise constant speed, and none of the parameters is turned on or off instantaneously. We further set the time spent in traversing each edge of the polygon equal to unity. This limitation could be relaxed, in which case the length of each edge in time would be an additional free parameter. We also set $C=1$ in Eq. (2). This can be achieved by properly fabricating the inductor, but we have every reason to believe that the algorithm will work for other choices of $C$ as well. Hence, in order to evaluate Eq. (4) one only needs to specify the coordinates of the vertices of the polygon, which we denote collectively as $X_{\gamma}$. Numerically, it is easy to evaluate the unitary operator in a stable manner by further dividing the loop $\gamma(t)$ into tiny intervals that take the time $\Delta t$ to traverse. If $\gamma_{i}$ denotes all the values of the parameters in the midpoint of the $i^{t h}$ interval, and $m$ is the number of such intervals, then we find to a good approximation

$$
U_{X_{\gamma}} \approx \exp \left(-i H\left(\gamma_{m}\right) \Delta t\right) \ldots \exp \left(-i H\left(\gamma_{1}\right) \Delta t\right) .
$$

We now proceed to transform the problem of finding the desired unitary operator into an optimization task. Namely, any $\hat{U}$ can be found as the solution of the problem of minimizing the error functional

$$
f\left(X_{\gamma}\right)=\left\|\hat{U}-U_{X_{\gamma}}\right\|_{F}
$$

over all possible values of $X_{\gamma}$. Here $\|\cdot\|_{F}$ is the Frobenius trace norm defined as $\|A\|_{F}=\sqrt{\operatorname{Tr}\left(A^{\dagger} A\right)}$. The number of adjustable vertices of the polygon $\nu$ is kept fixed from the beginning. One needs to have enough vertices to parameterize the unitary group $S U\left(2^{N}\right)$. The dimension of this group is $2^{2 N}-1$ and there are $2 N$ parameters for each vertex. Thus, we must have $2 N \nu \geq 2^{2 N}-1$. We 
use $\nu=12$ for the three-qubit gates and $\nu=4$ for the two-qubit gates. Within this formulation the method of finding the desired gates is similar to the recently introduced method of finding holonomic quantum gates [1]. Thus we again expect the minimization landscape to be rough and we apply the robust polytope algorithm [16] for the minimization.

We concentrate on finding two- and three-qubit gates, since one-qubit gates can be trivially constructed with the help of Euler angles. A larger quantum gate could be performed by factoring it into two- and three-qubit operations, and the implementation for these could be found numerically. It seems that quantum operations for four, five or more qubits could be found with the same method, assuming that sufficient computing resources are available. However, even in the case of three-qubit gates the optimization task becomes challenging and we need to use parallel programming. In the parallel three-qubit program, since the function evaluations of $f\left(X_{\gamma}\right)$ require a major part of the computation, we distribute the workload such that each processor calculates the contribution of a single edge of the polygon. In addition, one processor handles the minimization routine.

Let us turn to the results. First, we attempt to construct a gate equivalent to the controlled-NOT, namely

$$
U=\exp \left(i \frac{\pi}{4}\right)\left[\begin{array}{llll}
1 & 0 & 0 & 0 \\
0 & 1 & 0 & 0 \\
0 & 0 & 0 & 1 \\
0 & 0 & 1 & 0
\end{array}\right]
$$

The phase factor is needed in order for the gate to belong to $S U(4)$. It is already hard to see from the form of the Hamiltonian how this gate would be carried out in the present setting. Figure 3 illustrates an implementation of this gate that has been obtained by minimizing the error function in Eq. (6); the error is negligible. This example clearly illustrates the potential of our method.

As a second example, we construct the two-qubit Fourier transform. The quantum Fourier transform (see e.g., Ref. [2]) is given in the case of two qubits by

$$
F_{2}=\frac{1}{2}\left[\begin{array}{cccc}
1 & 1 & 1 & 1 \\
1 & i & -1 & -i \\
1 & -1 & 1 & -1 \\
1 & -i & -1 & i
\end{array}\right]
$$

Furthermore, we need to multiply this by $\exp \left(i \frac{\pi}{8}\right)$ in order to find a gate that belongs to $S U(4)$. Figure 4 shows the resulting loop that has been found with the help of the algorithm. In general, the optimization task for two-qubit gates can be performed quite easily with the help of personal computers. However, finding threequbit gates is already quite time-consuming. It proves worth the extra effort to do this, though.
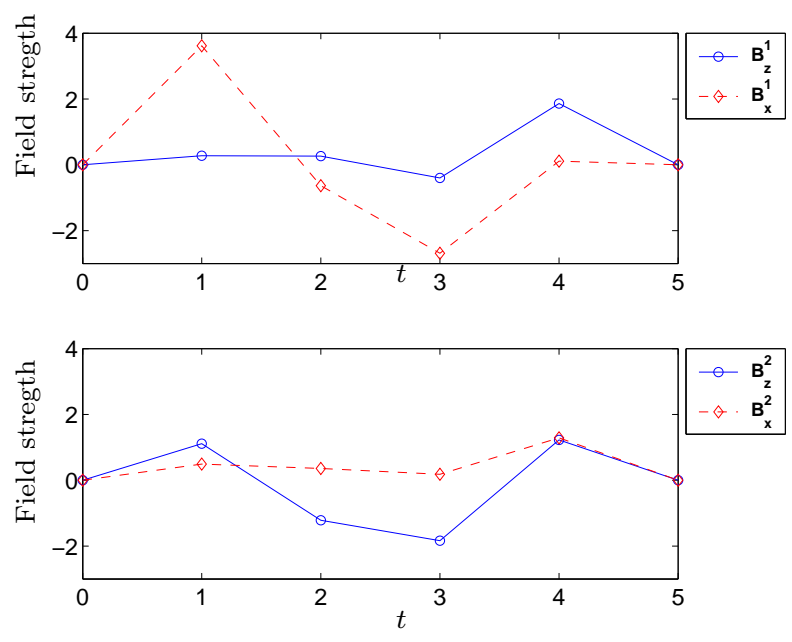

FIG. 3: Control-parameter sequences as functions of time that yield the gate in Eq. (7) which is equivalent to the controlled-NOT. The relative error is on the order of $10^{-11}$ and 100 discretization points per edge were used.
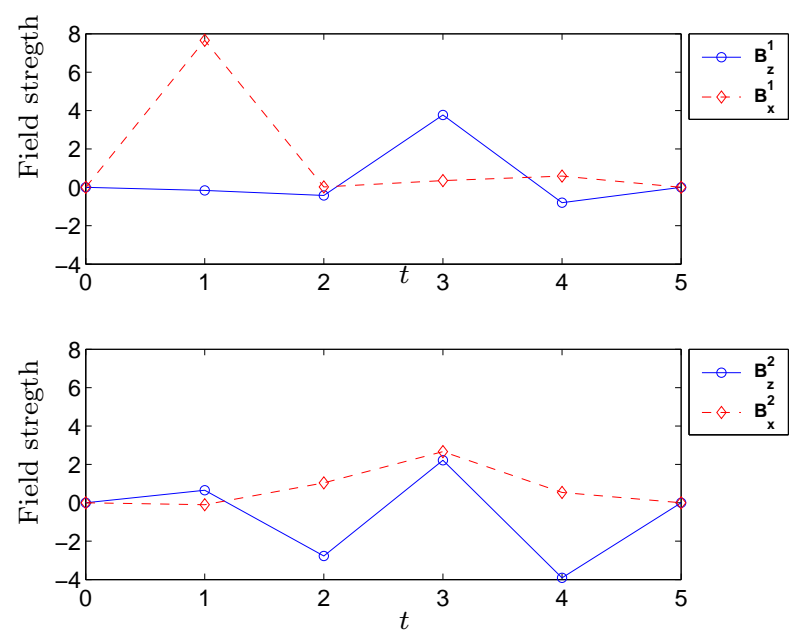

FIG. 4: Control-parameter sequences as functions of time that yield the two-qubit Fourier transform in Eq. (8). The relative error is on the order of $10^{-11}$ and 100 discretization points per edge were used.

The three-qubit quantum Fourier transform is [2]

$$
F_{3}=\frac{1}{\sqrt{8}}\left[\begin{array}{cccccccc}
1 & 1 & 1 & 1 & 1 & 1 & 1 & 1 \\
1 & \omega & \omega^{2} & \omega^{3} & \omega^{4} & \omega^{5} & \omega^{6} & \omega^{7} \\
1 & \omega^{2} & \omega^{4} & \omega^{6} & 1 & \omega^{2} & \omega^{4} & \omega^{6} \\
1 & \omega^{3} & \omega^{6} & \omega^{6} & \omega^{4} & \omega^{7} & \omega^{2} & \omega^{5} \\
1 & \omega^{4} & 1 & \omega^{4} & 1 & \omega^{4} & 1 & \omega^{4} \\
1 & \omega^{5} & \omega^{2} & \omega^{7} & \omega^{4} & \omega^{1} & \omega^{6} & \omega^{3} \\
1 & \omega^{6} & \omega^{4} & \omega^{2} & 1 & \omega^{6} & \omega^{4} & \omega^{2} \\
1 & \omega^{7} & \omega^{6} & \omega^{5} & \omega^{4} & \omega^{3} & \omega^{2} & \omega
\end{array}\right]
$$

where $\omega=\exp \left(i \frac{\pi}{4}\right)$. Since $\operatorname{det}\left(F_{3}\right)=i$ we must set $\hat{U}=\exp \left(-i \frac{\pi}{16}\right) F_{3}$ such that $\hat{U} \in S U(8)$. As an evidence of the success of the three-qubit algorithm, we have in Fig. 5 plotted the implementation of the threequbit Fourier transform. We conclude from these three 

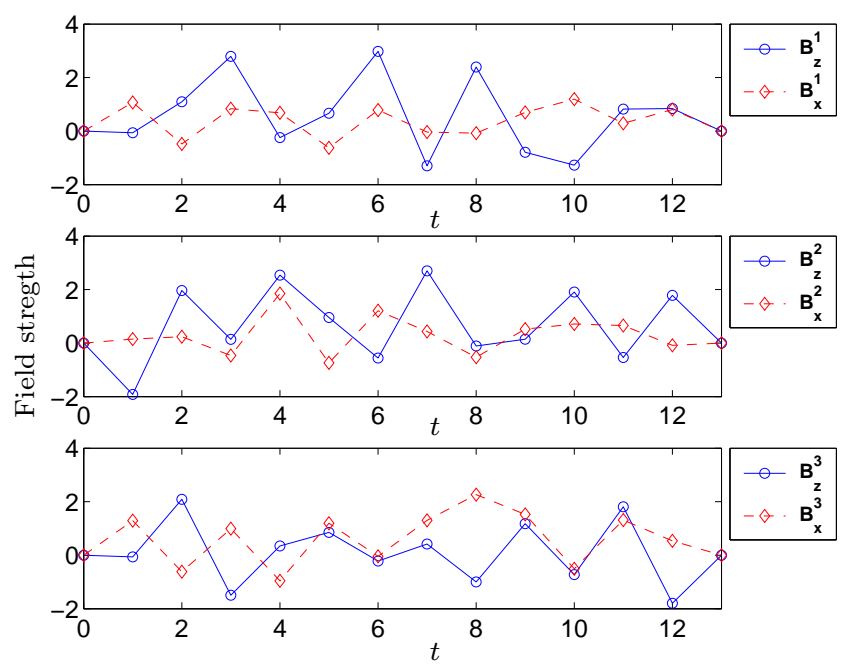

FIG. 5: Control-parameter sequences as functions of time that yield the three-qubit quantum Fourier transform (modulo a global phase). The relative error is on the order of $10^{-5}$ and 100 discretization points were used.

examples that it is possible to find far more powerful optimal implementations of multiqubit quantum gates with the help of the minimization scheme.

To further assess the strength of the technique, we compare the number of steps that are required to carry out the three-qubit Fourier transform using only twoqubit gates with the number of steps required when using the full three-qubit implementation of Fig. 5. The two-qubit implementation [18] requires effectively four gates, see Fig. 11 Since these gates would have to be performed sequentially, we would need five polygon edges per two-qubit operation. This results in 20 edges for the whole operation. Using elementary gates would require far more edges. Our optimized three-qubit Fourier transform, though, only requires 13 edges. Since each edge contributes the same amount to the operation time, we conclude that our implementation is improved. What is more, not all multiqubit gates can be decomposed as conveniently as the Fourier transform. For them the gain is higher. Thus, increasing the amount of classical computing resources should yield even better results.

In conclusion, we have described how to efficiently construct two- and three-qubit quantum gates for the Josephson charge qubit using numerical optimization. An immediate strength of the present scenario is that one avoids unnecessary idle time during the logical quantum operations. Since the loops are traversed at a piecewise constant speed, and no fields are instantaneously switched, this method of constructing quantum gates should be viable from the experimental point of view as well. The effect of finite fall and rise times of pulses on the quality of quantum gates has been studied recently 17]. Since we do not use pulses but instead interpolate along linear paths in the parameter space, such errors can be avoided. It seems reasonable to construct large-scale quantum algorithms in multiqubit blocks. This can be accomplished by optimizing the gate realization with the help of classical computers.

The authors would like to thank M. Nakahara for useful discussions. AON and JJV thank the Research Foundation of Helsinki University of Technology and the Graduate School in Technical Physics for financial support; this work is supported by the Academy of Finland through a Research Grant in Theoretical Materials Physics. We also thank the Center for Scientific Computing (CSC, Finland) for computing resources.

* Currently at VTT Information Technology, Microsensing, POB 1207, 02044 VTT, Finland; Electronic address: antti.niskanen@vtt.fi

† Electronic address: juhav@focus.hut.fi

[1] A. Barenco, C. H. Bennett, R. Cleve, D. P. DiVincenzo, N. Margolus, P. Shor, T. Sleator, J. Smolin, and H. Weinfurter, Phys. Rev. A 52, 3457 (1995).

[2] J. Gruska, Quantum Computing, McGraw-Hill, New York (1999); M. A. Nielsen and I.L. Chuang, Quantum Computation and Quantum Information, Cambridge University Press, Cambridge (2000); A. Galindo and M. A. Martin-Delgado, Rev. Mod. Phys. 74, 347 (2002).

[3] A. Shnirman, G. Schön, and Z. Hermon, Phys. Rev. Lett. 79, 2371 (1997); D. V. Averin, Solid State Commun. 105, 659 (1998).

[4] Yu. Makhlin, G. Schön, and A. Shnirman, Rev. Mod. Phys. 73, 357 (2001).

[5] J. Q. You, J. S. Tsai, and F. Nori, Phys. Rev. Lett. 89, 197902 (2002).

[6] W. H. Zurek, Rev. Mod. Phys. 75 (in print, 2003).

[7] Y. Nakamura, Yu. A. Pashkin, and J. S. Tsai, Nature 398, 786 (1999); Phys. Rev. Lett. 87, 246601 (2001).

[8] T. P. Orlando, J. E. Mooij, L. Tian, C. H. van der Wal, L. Levitov, S. Lloyd, and J. J. Mazo, Phys. Rev. B 60, 15398 (1999).

[9] J. M. Martinis, S. Nam, J. Aumentado, and C. Urbina, Phys. Rev. Lett. 89, 117901 (2002); Y. Yu, S. Han, X. Chu, S. Chu, and Z. Wang, Science 296, 889 (2002).

[10] D. Vion, A. Aassime, A. Cottet, P. Joyez, H. Pothier, C. Urbina, D. Esteve, and M. H. Devoret, Science 296, 886 (2002).

[11] A. O. Niskanen, M. Nakahara, and M. M. Salomaa, Quantum Information and Computation 2, 560 (2002); Phys. Rev. A 67, 012319 (2003).

[12] P. Zanardi and M. Rasetti, Phys. Lett. A 264, 94 (1999).

[13] J. Siewert and R. Fazio, Phys. Rev. Lett. 87, 257905 (2001).

[14] J. P. Palao and R. Kosloff, Phys. Rev. Lett. 89, 188301 (2002).

[15] X. Wang, A. Sørensen, and K. Mølmer, Phys. Rev. Lett. 86, 3907 (2001).

[16] J. C. Lagarias, J. A. Reeds, M. H. Wright, and P. E. Wright, SIAM J. Optim. 9, 112 (1998).

[17] S. Oh, Phys. Rev. B 65, 144526 (2002).

[18] We assume that one-qubit operations are embedded into two-qubit gates. 